\title{
Heterogenität schizophrener Psychosen im Langzeitverlauf - eine Herausforderung für klinische Praxis und Forschung
}

\author{
Heterogeneity of Schizophrenic Psychoses in the Long-Term Course - \\ A Challenge for Clinical Practice and Research
}

Autor

Institut
Markus Jäger

Klinik für Psychiatrie und Psychotherapie II der Universität Ulm, Bezirkskrankenhaus Günzburg

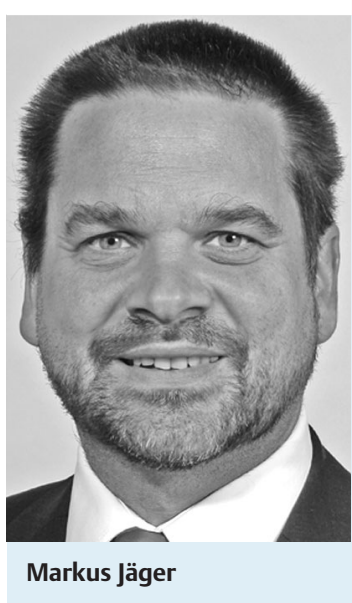

Bibliografie

DOI http://dx.doi.org/

10.1055/s-0034-1370096

Psychiat Prax 2014; 41:

291-292

(c) Georg Thieme Verlag KG Stuttgart · New York

ISSN 0303-4259

Korrespondenzadresse Prof. Dr. med. Markus Jäger

Klinik für Psychiatrie und

Psychotherapie II

der Universität Ulm,

Bezirkskrankenhaus Günzburg

Ludwig-Heilmeyer-Straße 2

89312 Günzburg

Markus.Jaeger@bkh-guenzburg.

de

\section{Schizophrenie als heterogene Erkrankungsgruppe \\ $\nabla$}

Als Eugen Bleuler 1911 den Begriff der Schizophrenie prägte, sprach er hierbei ausdrücklich von der „Gruppe der Schizophrenien“ und brachte auf diese Weise die Heterogenität der Erkrankungsgruppe klar zum Ausdruck [1]. Ein historischer Rückblick macht deutlich, dass es sich beim Schizophreniebegriff keineswegs um ein einheitliches nosologisches Konstrukt handelt, sondern dass es hier eine Reihe von zum Teil sehr unterschiedlichen Konzepten gibt [2]. Mithilfe der modernen kriterienorientierten Diagnosesysteme konnte inzwischen eine gewisse begriffliche Konventionalisierung erzielt werden, welche die Durchführung und die Vergleichbarkeit von empirischen Studien erheblich erleichtert.

Die schizophrenen Psychosen stellen jedoch weiterhin eine recht heterogene Erkrankungsgruppe dar, insbesondere auch im Hinblick auf die psychopathologische Symptomatik und den Verlauf. Dies wird auch durch neuere empirische Studien bestätigt. Selbst wenn moderne kriterienorientierte Diagnosesysteme verwendet werden, weisen die schizophrenen Psychosen eine erhebliche Verlaufsvarianz auf. So liegen beispielsweise die klinischen Remissionsraten bei Patienten mit der Diagnose einer Schizophrenie (Kriterien gemäß DSM-III, DSM-III-R, DSM-IV oder ICD-10) nach einem Verlauf von mindestens 5 Jahren in einem weiten Spektrum zwischen 7\% und 52\% [3].

\section{Einflussfaktoren auf den Langzeitverlauf $\nabla$}

Zahlreiche empirische Studien haben sich darum bemüht, die Verlaufsvarianz schizophrener Psychosen mithilfe von statistischen Modellen aufzuklären und entsprechende Prädiktoren zu identifizieren. Die meisten dieser Untersuchungen kamen zu dem Ergebnis, dass Faktoren wie ausge- prägte Negativsymptomatik in einem frühen Krankheitsstadium, männliches Geschlecht, kognitive Störungen, niedriges Bildungsniveau, soziale Isolation und eine lange Dauer der unbehandelten Psychose (DUP) auf einen ungünstigen Verlauf hinweisen [3]. Jedoch kann durch diese Prädiktoren zumeist nur ein eher geringer Teil der Gesamtvarianz erklärt werden.

In vielen Studien wurde deutlich, dass die psychopathologische Symptomatik im Langzeitverlauf schizophrener Psychosen eine deutliche Stabilität aufweist [3]. Hieran anschließend stellt sich die entscheidende Frage, ob der Verlauf schizophrener Psychosen bereits bei Ausbruch der Erkrankung im Wesentlichen neurobiologisch präformiert ist, oder ob der Verlauf eher von äußeren Einflussfaktoren abhängt. Insbesondere ist hierbei auch unklar, inwiefern der Langzeitverlauf nachhaltig durch therapeutische Maßnahmen beeinflusst werden kann.

\section{Dimensionale Erfassung oder typo- logische Ordnung $\nabla$}

Aufgrund von Heterogenität und fehlender Validierung durch neurobiologische Befunde wird das nosologische Konstrukt der Schizophrenie immer wieder infrage gestellt [2]. Als Alternative zum aktuellen Schizophreniekonzept wird häufig ein dimensionaler Ansatz vorgeschlagen. Hierbei wird versucht, die nosologische Einheit der Schizophrenie in verschiedene Symptomdimension aufzulösen wie beispielsweise Positivsymptomatik, Negativsymptomatik, Desorganisation und Depression [4]. Durch ein solches dimensionales Modell ist eine differenzierte Erfassung der psychopathologischen Querschnittssymptomatik möglich. Dieser Ansatz ist jedoch mit der Gefahr verbunden, dass die verschiedenen im Krankheitsverlauf auftretenden Symptome unverbunden nebeneinander aufgelistet werden, ohne den 
Verlaufsaspekt ausreichend zu berücksichtigen. So dürfte es eher schwerfallen, mit einem rein dimensionalen Modell typische Verlaufsmuster schizophrener Psychosen anschaulich abzubilden und einzuordnen.

Die Erarbeitung einer differenzierten Subtypologie schizophrener Psychosen kann deshalb eine Alternative - oder besser gesagt eine Ergänzung - zu einem dimensionalen Ansatz darstellen [2]. Hierbei sollte man sich darum bemühen, psychopathologische Verlaufscharakteristika anschaulich herauszuarbeiten. Der empirische Befund einer hohen Verlaufsstabilität der psychopathologischen Symptomatik bei schizophrenen Psychosen kann als unterstützender Beleg für einen solchen Ansatz angesehen werden [3]. Allerdings gibt es bisher keine allgemein anerkannte psychopathologische Verlaufstypologie schizophrener Psychosen, die sowohl ausreichend empirisch validiert als auch problemlos in der Praxis anwendbar ist. Die traditionelle Unterteilung schizophrener Psychosen in paranoide, katatone und hebephrene Formen hat sich nur als bedingt hilfreich erwiesen, sodass beispielsweise im DSM-5 auf diesen Ansatz verzichtet wird [5]. Die Etablierung einer alternativen Typologie stellt deshalb eine wichtige Aufgabe der aktuellen psychiatrischen Forschung dar. Hierbei sollte man eine quantitativ-statistische Analyse mit einer kasuistischen Vorgehensweise verbinden [6].

\section{Bedeutung für die klinische Praxis \\ $\nabla$}

Aufgrund der Heterogenität der schizophrenen Psychosen ist im Einzelfall eine differenzierte Betrachtung erforderlich. Für die klinischen Entscheidungen bildet die Diagnose einer schizophrenen Psychose alleine in den meisten Fällen keine ausreichende Grundlage. Die Kenntnis von regelmäßig vorkommenden psychopathologischen Verlaufstypen schizophrener Psychosen ist mehr noch als die dimensionale Erfassung der Querschnittssymptomatik in der klinischen Praxis von Bedeutung. Eine Verlaufstypologie und die diagnostische Einordnung der individuellen Patienten in eine solche Typologie kann die Grundlage für viele klinische Entscheidungsprozesse sein. Hier sind beispielsweise Wahl der Pharmakotherapie, Art der Psychoedukation, Planung rehabilitativer Maßnahmen und gutachterliche Äußerungen zu nennen.

Allerdings sollte man vorsichtig sein, bei einem Patienten vorschnell einen ungünstigen Verlaufstyp anzunehmen, da dies zu einem ungerechtfertigten Pessimismus bis hin zu einer sich selbst erfüllenden Prophezeiung führen kann [7]. Die Annahme eines ungünstigen Verlaufstyps muss jedoch nicht zwangsläufig zu einem therapeutischen Nihilismus führen. Auch schwere und chronische Verlaufsformen schizophrener Psychosen können durchaus mithilfe von angemessenen Interventionen positiv beeinflusst werden. Eine solche typologische Zuordnung könnte auch helfen, im Einzelfall realistische Therapieziele zu formulieren. Geht man nämlich bei einem Patienten von einer zu günstigen Verlaufsprognose aus, kann dies zu einer Überforderung der Betroffenen führen, welche dann möglicherweise selbst wieder einen negativen Einfluss auf den weiteren Krankheitsverlauf hat. Beachtet man das Auftreten von zum Teil recht unterschiedlichen Verlaufstypen schizophrener Psychosen, lässt sich auch die Diskussion um die Konzepte „Chronizität“ und „Recovery“ deutlich versachlichen [7].

\section{Bedeutung für die Forschung}

Eine differenzierte psychopathologische Verlaufstypologie schizophrener Psychosen könnte nicht nur in der klinischen Praxis, sondern auch für die künftige psychiatrische Forschung von Bedeutung sein. Bisher ist es nicht gelungen, die traditionellen nosologischen Konstrukte in der Psychiatrie wie beispielsweise die Schizophrenie durch neurobiologische Befunde zu validieren. Einen solchen Ansatz kann man natürlich grundsätzlich infrage stellen. Auf der anderen Seite lässt sich aber auch die Ansicht vertreten, dass die bisherigen nosologischen Konstrukte zu heterogen waren, und deshalb eine Korrelation mit neurobiologischen Befunden zu keinen wesentlichen Ergebnissen geführt hat.

Seit einigen Jahren wird deshalb zunehmend das Konzept verfolgt, verschiedene psychopathologische Symptome als Ausdruck einer Störung von unterschiedlichen funktionellen Systemen im Gehirn anzusehen [8, 9]. Hieraus könnten dann Beeinträchtigungen in den Domänen „Sprache“, „Affekte“ oder „Körperbewegung“ resultieren [9]. Verschiedene Verlaufstypen schizophrener Psychosen könnten in einem solchen Forschungsansatz mit unterschiedlichen funktionellen Systemen in Verbindung gesetzt werden. Weiterhin besteht die Möglichkeit, die einzelnen Verlaufstypen mit den Ergebnissen molekularbiologischer Untersuchungen zu korrelieren, wobei hier jedoch große Fallzahlen erforderlich sind. Ein solcher Ansatz wird gegenwärtig in Deutschland mit der sogenannten „DGPPN-Kohorte“ verfolgt [10].

\section{Literatur}

1 Bleuler E. Dementia praecox oder die Gruppe der Schizophrenien. In: Aschaffenburg G, Hrsg. Handbuch der Psychiatrie. Teil 4. Leipzig: Deuticke; 1911

2 Jäger M, Frasch K, Lang FU et al. Auflösung des Schizophreniebegriffes Dimensionale Modelle oder Aufteilung in Subtypen? Nervenarzt 2012; 83: $345-354$

3 Lang FU, Kösters M, Lang S et al. Psychopathological long-term outcome of schizophrenia - a review. Acta Psychiatr Scand 2013; 127: 173 -182

4 Häfner $H$. Ist die Diagnose Schizophrenie noch sinnvoll? Psychiat Prax 2007; 34: 175 - 180

5 American Psychiatric Association. Diagnostic and Statistical Manual of Mental Disorders. Fifth Edition. Arlington: American Psychiatric Association; 2013

6 Jäger $M$, Weiser $P$, Becker $T$ et al. Identification of psychopathological course trajectories in schizophrenia. Psychiatry Res 2014; 215: $294-$ 299

7 Amering $M$, Bottlender $R$. Das Konzept der Chronizität psychischer Erkrankungen ist aufzugeben. Psychiat Prax 2009; 36: 4-6

8 Gaebel $W$, Wölwer W, Zielaseck J. Von der deskriptiven zur funktionalen Psychopathologie. Auf dem Weg zu einer modularen Psychiatrie. Die Psychiatrie 2006; 3: 221-232

9 Strik W, Dierks T. Biologische Psychopathologie. Stuttgart: Kohlhammer; 2011

10 Anderson-Schmidt H, Adler L, Aly C et al. The "DGPPN-Cohort" - a national collaboration initiative by the German Association for Psychiatry and Psychotherapy (DGPPN) for establishing a large-scale cohort of psychiatric patients. Eur Arch Psychiatry Clin Neurosc 2013; 263: 695-701 